\title{
Evolución tectónica del sector de Lorca desde el Mioceno superior al Cuaternario
}

\author{
CARLOS SANZ DE GALDEANO ${ }^{1}$, JOAQUÍN GARCÍA-MONDÉJAR ${ }^{2}$ \& \\ JOSE MARÍA FERRAIRÓ ${ }^{3}$ \\ ${ }^{1}$ Instituto Andaluz de Ciencias de la Tierra (CSIC- Univ. Granada). Facultad de Ciencias. 18071, Granada \\ ${ }^{2}$ Dpto. de Estratigrafía y Paleontología, Facultad de Ciencia y Tecnología, Universidad del País Vasco, \\ UPV/EHU, apartado 644, 48080, Bilbao. \\ ${ }^{3}$ Dpto. Ciencias Naturales. I.E.S. "Ausiàs March". 46702, Gandía (Valencia) \\ csanz@ugr.es
}

Recibido: $18 / 04 / 2012$

Aceptado: 03/10/2012

\begin{abstract}
Resumen
La falla de Alhama de Murcia presenta diversas superficies que forman una amplia zona de falla. Incluso a distancias de varios kilómetros hay otras fallas paralelas que pertenecen al mismo juego. Estas fallas crearon estrcturas subsidiarias, algunas de tamaños kilométricos. Así, en el sector de Lorca se levantó progresivamente, desde finales del Mioceno medio, el sector de Peña Rubia. Más adelante, y en parte coetáneamente, se formó el antiforme del Castillo. El movimiento sinistrorso de la gran falla de Alhama de Murcia, y el de sus conjugadas dextrorsas fue provocado por un esfuerzo que correspondió a una compresión casi N-S y una extensión perpendicular ligada. También se detectan algunos momentos en los que se produjo un intercambio entre las posiciones de los ejes $\sigma_{1}$ y $\sigma_{2}$, lo que permitió, particularmente en fallas de dirección aproximada N-S, desplazamientos de tipo normal y por tanto la posibilidad de ese tipo de mecanismos focales en algunos terremotos. El borde NE de Peña Rubia (en el extremo oriental de la sierra de las Estancias), que a su vez constituye el borde SE de la cuenca de Lorca, está afectado por fallas menores de salto en dirección e inversas igualmente coherentes con el paleoesfuerzo deducido. Sin embargo, a lo largo de ese borde no se observa ninguna falla continua e importante.
\end{abstract}

Palabras clave: Neotectónica, falla de desgarre, paleoesfuerzos, Neógeno.

\section{Tectonic evolution of the Lorca sector from the Late Miocene to the Quaternary}

\begin{abstract}
The Alhama de Murcia Fault presents several parallel surfaces, forming a wide fault zone. Even at distances of several $\mathrm{km}$, there are other faults belonging to the same system. These faults created subsidiary structures, some of kilometric lengths, as in the case of the progressive uplift of the Peña Rubia sector, occurring from the end of the Middle Miocene. Later and partially coeval, was the formation of the Castillo antiform. The sinistral displacement of the large Alhama de Murcia Fault and that of smaller dextral conjugated faults was caused by stress from a nearly N-S compression and a related perpendicular extension. At some points in time, the positions of $\sigma_{1}$ and $\sigma_{2}$ axes switched, permitting normal movements, particularly in faults with approximately N-S directions, and hence raising the possibility of this type of focal mechanism in some earthquakes.The NE border of Peña Rubia (on the eastern end of Sierra de Las Estancias), also the SE border of the Lorca Basin, is affected by small (compared with the main ones) strike-slip and reverse faults that are also consistent with the palaeostress deduced. Nevertheless, along this border no significant or continuous fault is detected.
\end{abstract}

Keywords. Neotectonics, strike-slip fault, paleostress, Neogene 


\section{Referencia normalizada}

Sanz de Galdeano, C., García Mondejar, J. y Ferrairó, J.M. (2012). Evolución tectónica del sector de Lorca desde el Mioceno superior al Cuaternario. Física de la Tierra, 24, 193-211.

Sumario: Introducción. 1. Encuadre geológico. 1.1. Principales rasgos estratigráficos del sector de Lorca 2. Tectónica. 2.1. Estudio de estructuras menores. 3. Discusión e interpretaciones. 4. Conclusiones. Agradecimientos. Referencias bibliográficas.

\section{Introducción}

Juegos de fallas importantes a escala regional suelen tener asociadas estructuras de menor entidad cuyo estudio complementa el conocimiento de la evolución geológica de un determinado sector. Este es el caso del presente artículo en lo referido al sector de Lorca.

Este sector se sitúa en la Zona Interna de la Cordillera Bética cuyos rasgos geológicos más importantes son: a) La superposición de unidades tectónicas (ocurrida anteriormente y que no es objeto de este estudio) y b) El conjunto de grandes fallas sinistrorsas que desde más al norte, al menos desde Murcia, se dirigen hacia el SO, hasta el mar de Alborán.

La falla principal en el sector de Lorca es la de Alhama de Murcia que también atraviesa las localidades de Totana-Lorca-Puerto Lumbreras (recibe también otros diversos nombres), muestra diversas líneas paralelas, cuyo número varía según el punto que se considere (Figs. 1 y 2). Ha sido estudiada desde mediados del siglo pasado (y ahora mucho más, sobre todo a partir del terremoto de mayo de 2011). Rey Pastor (1951) la llamó Falla del Sangonera (otra denominación del río Guadalentín); la extendía desde Puerto Lumbreras hasta Callosa de Segura. Bousquet y Montenat (1974), Bousquet (1979) y Kampschuur et al. (1981) indicaron su carácter sinistrorso. Larouzière et al (1988) integraron este conjunto de fallas en la zona de Cizalla Trans-Alborán, que en su segmento Bético da lugar a una alineación de fracturas de desgarre litosféricas sinistrosas que va desde Almería a Alicante.

Martínez Díaz y Hernández Enrile (1991) determinaron varias fases de movimiento en la Falla de Alhama de Murcia (que abreviadamente llaman F.A.M.) debido a que la compresión máxima horizontal varió a lo largo del tiempo, desde la dirección N150E a la NNE-SSO con diversas oscilaciones. Tomaremos esa abreviación, sin puntos, para nombrar esta gran falla, entendiendo que se prolonga desde el sector de Murcia hasta el sur de Puerto Lumbreras, aunque sea netamente divisible en diversos segmentos (Silva et al., 1992 y 2003; Martínez-Díaz et al., 2012).

Booth y Azañón (2003) señalaron la existencia de discordancias en el Mioceno superior en la Sierra de la Tercia, en las proximidades de Totana, en un marco geológico parecido al ahora estudiado. Finalmente, García Mayordomo (2005) indica que la FAM comenzó a desarrollarse ya en el Serravalliense (Mioceno medio) y, a partir del Tortoniense, se formaron también fallas de dirección NO-SE. 
El presente artículo estudia la FAM a su paso por Lorca y especialmente las estructuras subordinadas que en ese sector se han formado a causa de sus movimientos. Estas estructuras se produjeron desde el Mioceno superior al Cuaternario. No estudia los últimos terremotos ni la paleosismicidad, temas de buena parte de los artículos del presente número

\section{Encuadre geológico}

La Cordillera Bética se divide fundamentalmente en Zona Interna y Zona Externa. La Zona Interna está formada por cuatro complejos tectónicos superpuestos que, de abajo arriba, son Nevado-Filábride, Alpujárride, Maláguide y la Dorsal. Los dos primeros presentan metamorfismo alpino que afecta desde materiales paleozoicos (y anteriores) a mesozoicos, sobre todo triásicos. El Maláguide abarca desde los términos prepaleozoicos hasta el Terciario. La Dorsal y la Zona Externa, esta última situada actualmente al norte de la Interna, están formadas por sedimentos mesozoicos y terciarios. La Zona Externa se divide en Prebético y Subbético de acuerdo con su posición y facies. El Subbético se encuentra cercano al sector de Lorca (Fig. 1).

La colisión oblicua de la Zona Interna (originalmente situada varios cientos de kilómetros más al E) con la Externa, a la que deformó profundamente, se produjo en el Mioceno inferior y los movimientos diferenciales entre ambas zonas se prolongaron durante el Mioceno medio (Sanz de Galdeano y Vera, 1992). Posteriormente, en el Mioceno superior, incluso quizás desde el Serravalliense, fue cuando se formaron las grandes fallas NNE-SSO a NE-SO que desde Alicante pasan por Murcia, Lorca, y llegan a Cabo de Gata (Almería) donde entran en el mar de Alborán. Y esas fallas, entre ellas la FAM, han prolongado sus movimientos hasta la actualidad. Y por la misma razón, las estructuras ligadas y los paleorrelieves que se formaron, además de las propias grandes fallas, también han evolucionado a lo largo de ese tiempo.

En el sector de Lorca están representados los complejos Alpujárride y Maláguide y varias unidades de carácter intermedio entre ambos complejos (tanto en lo referido a las series estratigráficas como al grado metamórfico). Sobre ellos se formaron, como en el resto de la cordillera, diversas cuencas neógenas. Al NO de Lorca se sitúa la cuenca del mismo nombre y directamente al E la cuenca del río Guadalentín. En conjunto, en las áreas deprimidas se localizan los sedimentos neógenos y en las altas afloran las unidades de la Zona Interna, alcanzando los sedimentos neógenos diversas alturas en las laderas 


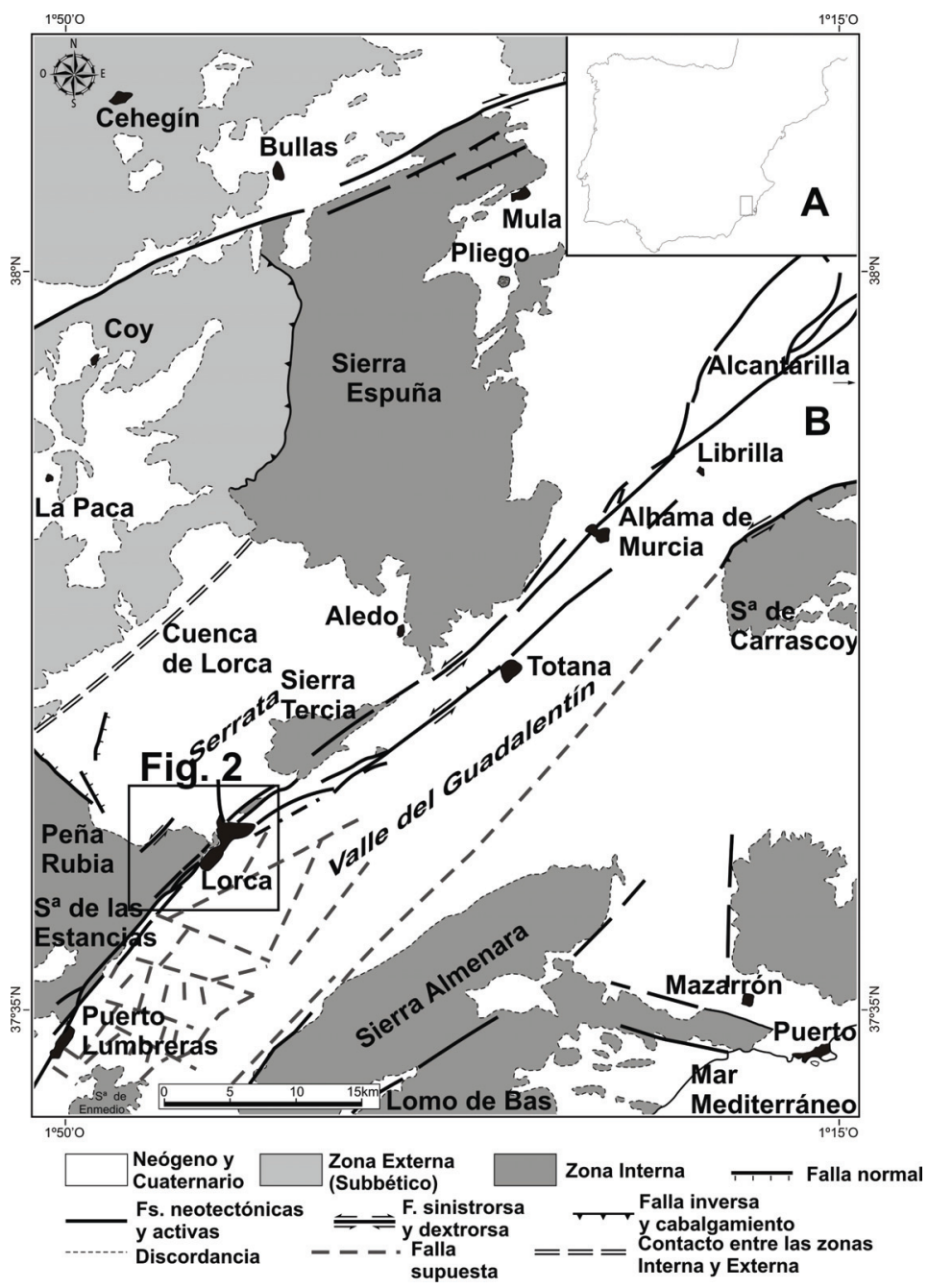

Fig. 1. Esquema geológico regional en el que se sitúa el área estudiada. Los datos de subsuelo de la Cuenca del Guadalentín se han deducido del IGME (1976) (sur de Lorca), y de Martínez Díaz (1998) y Montenat et al. (1990) (este de Lorca). Se indica la posición de la figura 2 .

\subsection{Principales rasgos estratigráficos del sector de Lorca}

Además del basamento bético formado por la Zona Interna, cuyas series abarcan aquí desde parte del Paleozoico al Triásico, afloran diversos depósitos neógenos y cuaternarios. Descripciones previas de los mismos se encuentran en Montenat et al. 
(1990), Pérez Lorente et al. (1992), Guillén-Mondéjar et al. (1995) y Booth Rea y Silva (2010). De forma sucinta y de techo a muro son los siguientes:

1. Lutitas con yesos de Cañada de Morales: Son discordantes sobre el basamento bético y afloran en diversos puntos del Castillo y del Barrio de San Cristóbal de Lorca. Están formados por areniscas y conglomerados intercalados entre lutitas y arcillitas verdes, grises y rosa salmón, con yesos, más abundantes a techo. Quizás correspondan al Langhiense.

2. Conglomerados rojos de Lorca: Afloran ampliamente en el Castillo y en el Barrio de San Cristóbal. Son los conglomerados poligénicos rojizos que reposan en discordancia sobre el sustrato bético o sobre los anteriores sedimentos. Localmente presentan evaporitas intercaladas. Su edad más probable es LanghienseSerravaliense (Booth Rea y Silva, 2010).

3. Margas y conglomerados grises del SE de Matalauva: Se componen de areniscas, conglomerados grises y margas amarillas con fauna marina, y presentan características de ambiente de transición fluvio-marino. Afloran en áreas del Castillo y al SE de Matalauva. Tienen una edad Langhiense-Serravaliense (Booth Rea y Silva, 2010).

4. Calizas del Castillo: Son calcarenitas de plataforma que representan el inicio de una importante transgresión. Pasan lateralmente hacia el norte a calcarenitas margosas y margas. Localmente se sitúan directamente sobre el basamento. Pérez-Lorente et al. (1992) les asignan una edad Tortoniense inferior y parte del superior.

5. Margas, calcarenitas y cherts del Llano de La Serrata: Son margas grises, calcarenitas y cherts que afloran extensamente en el Llano de La Serrata. Su base se indenta con las calizas infrayacentes y su parte superior es el equivalente temporal a los conglomerados tortonienses y messinienses. Corresponden al Tortoniense medio y superior, y quizás también al Messiniense inferior (Dinarès-Turell et al., 1998, y Rouchy et al., 1998).

6. Conglomerados del Campus Universitario: Son los conglomerados violáceos espesos de Montenat et al. (1990), quienes les atribuyen una edad Tortoniense II y un origen de abanico

Aluvial.

7. Calizas y conglomerados del repetidor de los Ángeles: Afloran al NE de Lorca. Se componen de calcarenitas con yesos y conglomerados aluviales. Booth Rea y Silva (2010) describen su unidad equivalente USD Va, que en parte corresponde en profundidad a halita y pizarras bituminosas (IGME, 1982). Por su equivalencia con las Fms. Nonihay y la Fm. de Aledo (Pérez Lorente et al., 1992), su edad es Messiniense inferior.

8. Yesos y margas de la entrada este de Lorca y La Serrata: Son yesos -detríticos y laminares- y margas, diversamente replegadas en proximidad de la FAM. y, en profundidad, halita y pizarras bituminosas (IGME, 1982). Se han atribuido al Messiniense inferior (Pérez Lorente et al., 1992) y al Messiniense superior (Booth Rea y Silva, 2010). Asociadas a las pizarras hay concentraciones notables de azufre, que fueron ampliamente explotadas, y cuya génesis debe estar ligada a la transforma- 
ción del sulfato de los yesos en azufre, gracias al ambiente reductor (o anóxico), de dicho depósito.

Además de esos materiales existen diversos tipos de depósitos coluviales y también travertinos, estos últimos aflorantes sobre todo al norte de Lorca, en los antiguos baños de Carraclaca, de diversas edades del Cuaternario (Martínez Díaz, 1998). También al sur de Lorca se ha encontrado un afloramiento muy pequeño de travertinos

\section{Tectónica}

La FAM tiene al norte de Lorca una dirección próxima a $\mathrm{N} 50^{\circ} \mathrm{E}$, pero precisamente desde Lorca hacia el SSO se produce una inflexión y pasa a ser N30E. La continuidad de las diferentes líneas de la falla al sur y al norte de Lorca no se observa bien, aunque pequeños afloramientos en distintas calles y datos del subsuelo obtenidos en solares excavados para construcción han permitido llevar las principales líneas de las fallas desde el SO hasta muy cerca de su prolongación por el NE (Fig. 2).

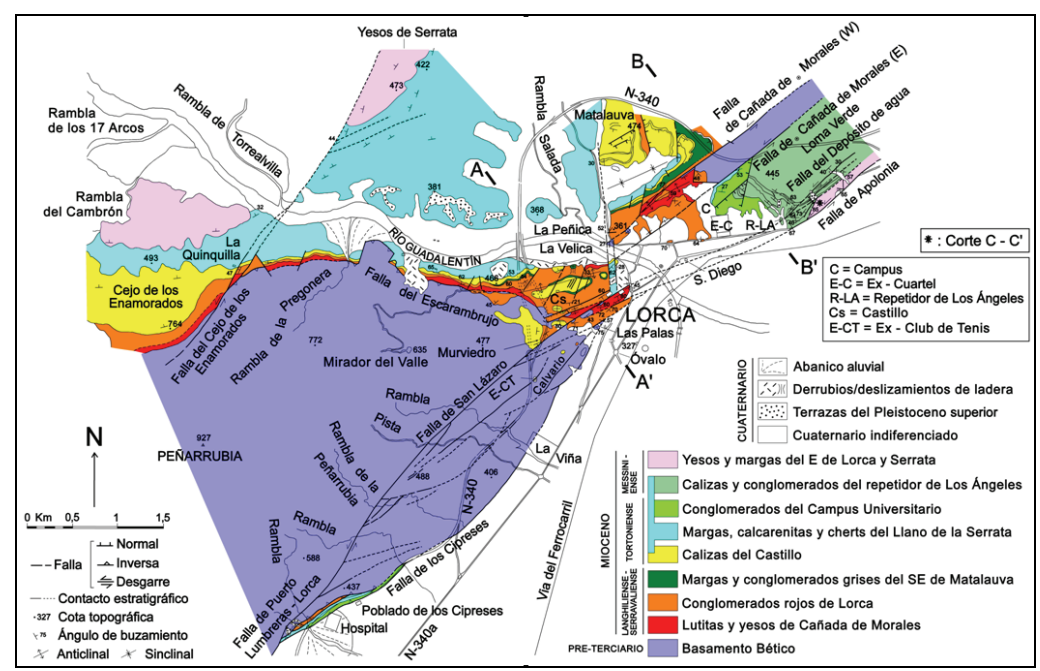

Fig 2. Mapa geológico del área de Lorca. Su posición se indica en la figura 1. Se marca la posición de los cortes de la figura 3 (A-A', B-B') y de la figura 4 (C-C').

Esas líneas de la FAM están aparentemente desplazadas unos 500m hacia el S al este del monte del Castillo. Es un desplazamiento de tipo sinistrorso. Se atribuye este desplazamiento a una falla que pasa al $\mathrm{N}$ y $\mathrm{NO}$ de Lorca, entre los lugares de la Velica y la Peñica, que muestra también una componente de deslizamiento vertical al norte del cauce del Guadalentín, hundiendo el labio occidental, de manera que sedimentos de los tramos $4^{\circ}$ y $5^{\circ}$, calizas y margas, se encuentran descendidos con respecto a otros más antiguos. Es una falla casi N-S que limita por el oeste los afloramientos de los tramos basales del Neógeno, calcarenitas incluidas, que vienen 
de Sierra Tercia. Su superficie no está bien expuesta, ni tampoco su continuidad hacia el norte es clara.

Estructuras existentes directamente al norte y noreste de Lorca en la FAM: Existen allí varias superficies de falla, en conjunto paralelas entre sí, con numerosas estrías horizontales, o casi, y crecimientos de fibras de yeso, además de estructuras S/C frágiles muy patentes. Esto ya fue descrito previamente (Bousquet y Montenat, 1974; Bousquet y Philip, 1976; Martínez Díaz, 1998, 1999, 2000, 2002; Martínez Díaz y Hernández Enrile, 1991, etc.). En su conjunto, la terminación SO de Sierra Tercia es un antiforme, cortado por las líneas de falla indicadas, en cuyo núcleo aflora en algunos puntos el basamento alpujárride, con materiales neógenos a ambos lados (en algunos casos no equivalentes entre sí) (Figs. 2 y $3 \mathrm{~A}$ ).

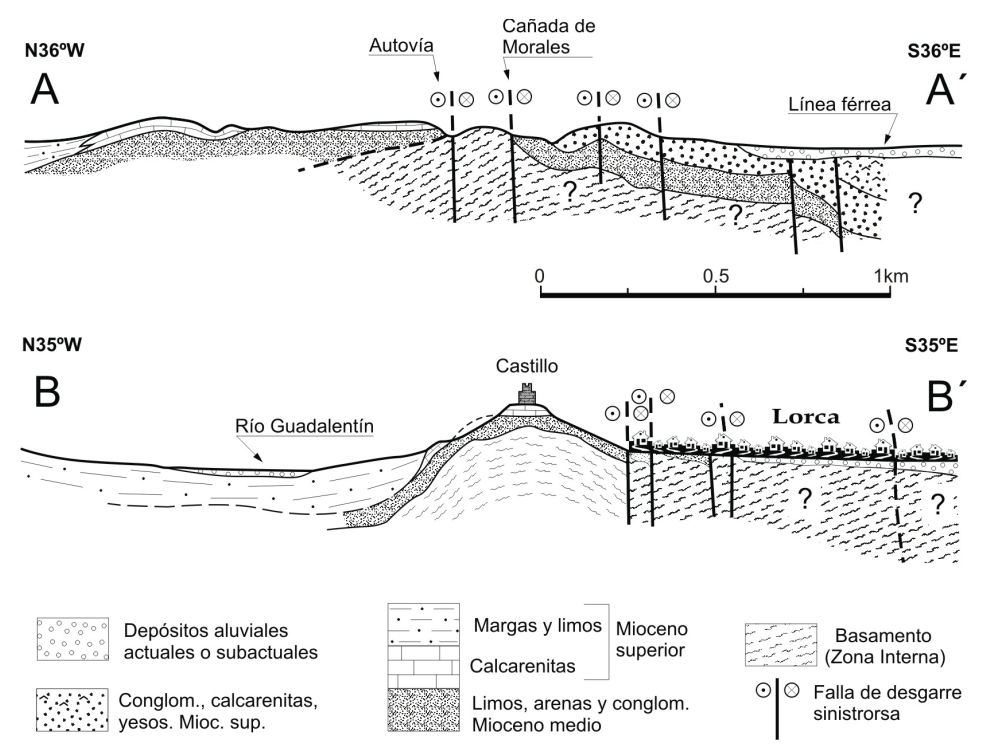

Fig 3. Cortes geológicos transversales a las principales estructuras. Su posición se indica en la figura 2

El corte de la Zona de Apolonia (Fig. 4) es un ejemplo de las estructuras de ese sector. Allí se observa una banda de unos $200 \mathrm{~m}$ de anchura en la que estratos del Messiniense inferior se han verticalizado y están cortados por varias líneas de falla, las principales de orientación $\mathrm{N} 50^{\circ} \mathrm{E}$. En ellas existen localmente estrías horizontales que indican netos desplazamientos sinistrorsos. La acción de las fallas también ha formado un pequeño antiforme bien visible en las proximidades del repetidor de Los Ángeles, con su eje buzante al SE. En estas estructuras existen numerosos pliegues asociados de ejes verticales, o casi, de tamaño métrico. También se obser- 
van enclaves tectónicos lenticulares de facies conglomeráticas entre calcarenitas (Fig. 4). Todo ello indica la transcurrencia sinistrorsa citada.

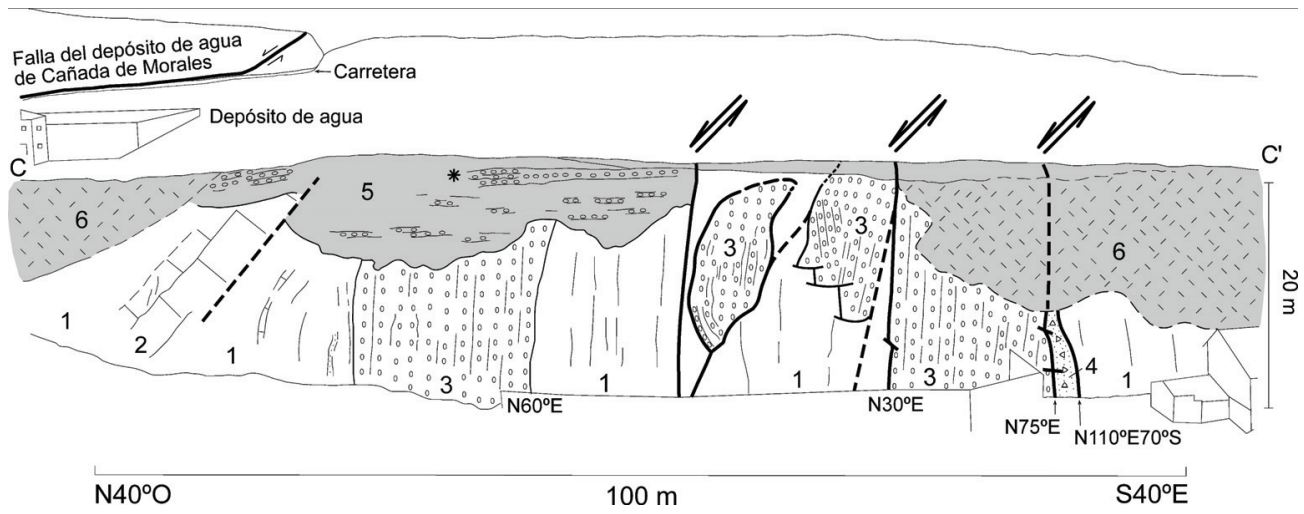

Fig 4.- Corte transversal parcial de la banda tectonizada del NE de Lorca (sector de Apolonia) elaborado a partir de fotografía. Materiales 1 a 4 : Messiniense inferior. 5 y 6: Pleistoceno superior. 1) Calcarenitas y margas con yesos; 2) Calcarenitas con cantos redondeados silíceos; 3) Conglomerados grises aluviales; 4) Brecha y conglomerado de matriz siliciclástica y calcárea; 5) Conglomerados de terraza aluvial (costra cementada en asteriscos); y 6) Derrubios de ladera. Las líneas gruesas corresponden a fallas. En el corte se indican direcciones de fallas y de estratos. Su posición se indica en la figura 2.

Estructuras existentes en el Castillo y hacia el sur: Entre el borde sur de la cuenca de Lorca (al O y NO de Lorca) y las fallas sobre las que se ubica el casco antiguo de la ciudad y su prolongación hacia el SSO se encuentra el sector del Castillo. Esta construcción se asienta sobre calcarenitas casi horizontales pero que hacia el norte toman muy fuerte buzamiento y hacia el ESE están cortadas y laminadas por varias de las líneas de la FAM, junto a tramos inferiores a las calcarenitas. Allí buzan en su conjunto hacia la FAM, es decir hacia el ESE. Por ello, la estructura del sector del Castillo es un anticlinal (Figs. 2 y 3B) que tiende a cerrarse al NE y que se va abriendo hacia el SO. En la parte occidental del castillo, en la pista que lo bordea, se ve bien la charnela anticlinal en los tramos inferiores a las calcarenitas.

El resto de la parte sur de Lorca está controlado por la FAM, allí con líneas de dirección $\mathrm{N} 30^{\circ}-50^{\circ} \mathrm{E}$ que al $\mathrm{SO}$ del hospital ya son $\mathrm{N} 30^{\circ} \mathrm{E}$. Estas líneas de falla controlan el cambio del relieve entre la sierra de las Estancias y el valle del Guadalentín (se da este nombre a la vega que existe directamente al E y NE de Lorca, aun cuando el río viene del O, sector que se denomina cuenca de Lorca).

Estructuras al oeste de Lorca: Se localiza allí la terminación septentrional de la Sierra de las Estancias (en ese punto se llama Sierra de Peña Rubia). Es una terminación brusca de dirección E-O. El límite es a la vez el del SE de la cuenca de 
Lorca. El cauce del Guadalentín pasa a poca distancia del dicho límite y de allí hacia el este llega a Lorca, donde su cauce se estrecha (Fig.2).

El contacto Sierra de las Estancias-Cuenca de Lorca parece, por tanto, propicio para situar una falla de dirección aproximada E-O, tal como hizo Martínez Díaz (1998), quien la llamó falla de las Viñas, nombre cogido de una agrupación de casas que hay varios kilómetros al oeste de Lorca (y que por tanto no hay que confundir con el barrio de La Viña muy maltratado en el terremoto del 11-05-2011). En este borde, los materiales neógenos buzan fuertemente hacia el norte y son localmente casi verticales. Este buzamiento es visible en los tramos inferiores a las calcarenitas, en estas mismas y en las margas superiores. En este sector, el espesor de los tramos inferiores es muy variable, donde pasan de tener más de $100 \mathrm{~m}$ a desaparecer a poca distancia; por ello, las calcarenitas se sedimentaron directamente sobre el basamento en bastantes puntos. La citada falla de las Viñas se situaría sobre todo a techo de las calcarenitas, y habría producido movimientos dextrorsos y normales. Sin embargo, el recorrido particularmente minucioso que se ha hecho de este borde permite descartar su existencia. Es un contacto estratigráfico en el que se ve bien el paso a las margas, a veces intercalando nuevas pasadas de calcarenitas. Sin embargo, ese contacto sí está cortado por numerosas fallas transversales de salto en dirección.

La observación de esas fallas transversales es interesante. Son fallas sinistrorsas paralelas a la FAM y dextrorsas, conjugadas con las anteriores, y en este caso de dirección general NO-SE a NNO-SSE. Ambos juegos muestran un neto predominio de estrías horizontales o casi. El desplazamiento de muchas de ellas se limita a unos pocos metros, pero en algunos casos rebasa el centenar de metros. Destaca particularmente la falla del sector del Cejo de los Enamorados que desplaza varios kilómetros hacia el sur al borde de la cuenca de Lorca.

En este mismo borde existen también pequeñas fallas inversas, prácticamente E$\mathrm{O}$, que se describen en el apartado de estructuras menores y una falla inversa mucho mayor que superpone materiales del basamento bético sobre los sedimentos neógenos a lo largo de más de $1 \mathrm{~km}$ (Fig. 2). Además, aproximadamente un $1 \mathrm{~km}$ al oeste del Castillo, la base de las calcarenitas está afectada por una falla paralela a la estratificación con movimientos casi horizontales sinistrorsos.

Estructuras en el valle del Guadalentín, al este de Lorca: En la figura 1 se indican a trazos diversas fallas cuya posición ha sido deducida a partir de datos de subsuelo (IGME, 1976). Solo se han situado algunas en las proximidades de Lorca, si bien existen trabajos que señalan su existencia tanto al $\mathrm{NE}$ como al SO en este valle como son los de Gauyau (1977) y Martínez Díaz (1998), entre otros. Como es obvio no son fallas visibles en superficie y se citan para que se tenga en cuenta el hecho de que el conjunto de las líneas de falla ligadas a la FAM es complejo y afecta a una zona aun más amplia que la que se observa en superficie. Y esas fallas también han tenido importancia en la evolución geológica de la zona, facilitando movimientos en horizontal y también en la vertical. De hecho, los cuerpos sedimentarios neógenos en las proximidades de Lorca muestran que, en algunos casos, el aporte de materiales venía de áreas actualmente erosionadas o bien hundidas tectónicamente. 


\subsection{Estudio de estructuras menores}

Son abundantes los puntos donde se pueden observar estriaciones en planos de falla, tanto en las grandes fallas como en las pequeñas. También hay numerosas estructuras frágiles del tipo $\mathrm{S} / \mathrm{C}$, crecimientos de fibras de yeso y de calcita y superficies con disoluciones estilolíticas, que si no se observan con cuidado pueden confundirse con estrías y dar sentidos de movimiento contrarios a los reales.

El sector donde se han obtenido más medidas se encuentra en Murviedro, al SO del Castillo (Fig. 2). Hay allí unas antenas de telefonía a cuyo pié existen varias canteras abandonadas que explotaron las calcarenitas. Existen en ellas espléndidos ejemplos de fallas sinistrorsas y dextrorsas conjugadas, con estriaciones horizontales o casi y cuyas direcciones medias en las sinistrorsas corresponden bien con la de la FAM. Allí el buzamiento de las calcarenitas varía entre 0 y $20^{\circ}$, si bien, fuera ya de ese sector, hacia la línea principal de la FAM, los buzamientos son mucho mayores.

Dado que el conjunto de esas medidas es bastante homogéneo, se han reunido todas para calcular los elipsoides de paleoesfuerzo. Para ello se ha utilizado el método de Galindo-Zaldívar y González Lodeiro (1988). El resultado indica la existencia de una fase principal y dos subfases. La más importante, a la que se asignan 32 fallas del total de 48, tiene la siguiente orientación: $\sigma_{1}=\mathrm{N} 162$, con un buzamiento de $4^{\circ}, \sigma_{2}=\mathrm{N} 342$ y buzamiento de $86^{\circ}$, y $\sigma_{3}=\mathrm{N} 72$, horizontal (Fig. 5 y tabla 1). Y la razón áxica $\left(\sigma_{2}-\sigma_{3} / \sigma_{1}-\sigma_{3}\right)$ es 0,39 , es decir, se trata de un elipsoide de esfuerzos prolato.

Tabla. 1 Paleoesfuerzos deducidos a partir de estructuras menores en los sectores de Murviedro y del $\mathrm{O}$ y $\mathrm{N}$ de Lorca

\begin{tabular}{|l|l|l|l|c|}
\hline \multicolumn{1}{|c|}{ Sector } & $\sigma_{1}$ & $\sigma_{2}$ & $\sigma_{3}$ & $\begin{array}{l}\text { Razón axial } \\
\left(\sigma_{2}-\sigma_{3} / \sigma_{1}-\sigma_{3}\right)\end{array}$ \\
\hline Murviedro Fase 1 & $\mathrm{N} 162 / 04$ & $\mathrm{~N} 342 / 86$ & $\mathrm{~N} 72 / 00$ & 0,39 \\
\hline Id., fase 2 (9 fallas) & $\mathrm{N} 174 / 50$ & $\mathrm{~N} 00 / 40$ & $\mathrm{~N} 267 / 03$ & 0,29 \\
\hline Id., fase 3 (6 fallas) & $\mathrm{N} 83 / 58$ & $\mathrm{~N} 332 / 12$ & $\mathrm{~N} 235 / 29$ & 0,25 \\
\hline O y N de Lorca & $\mathrm{N} 02 / 02$ & $\mathrm{~N} 106 / 82$ & $\mathrm{~N} 272 / 08$ & 0,36 \\
\hline
\end{tabular}

Además de esa fase hay otras dos de menor importancia (Fig. 5 y tabla 1), con pocas fallas asignadas, 9 y 6 respectivamente, cuyos elipsoides de esfuerzos son: para la que recibe 9 fallas para su cálculo, $\sigma_{1}=\mathrm{N} 174$, con un buzamiento de $50^{\circ}$, $\sigma_{2}=\mathrm{N} 0^{\circ}$ y buzamiento de $40^{\circ}$, y $\sigma_{3}=\mathrm{N} 267$ y $3^{\circ}$ de buzamiento. La razón áxica es 0,29 ; se trata de un elipsoide de esfuerzos prolato aún más marcado que el anterior. 


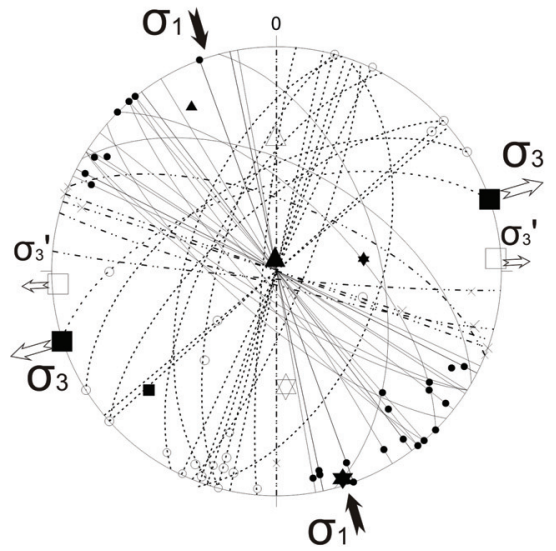

Murviedro

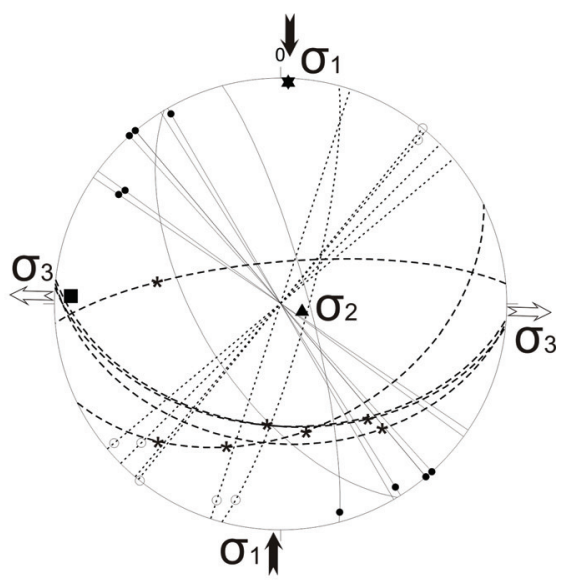

O y N de Lorca

Fig 5. Representación equiareal en el hemisferio inferior de las fallas medidas en el sector de Murviedro y $\mathrm{O}$ y $\mathrm{N}$ de Lorca. Las fallas dextrorsas se representan en línea continua (y la posición de las estrías se indica con un punto negro); las sinistrorsas a trazos (las estrías con un círculo). En Murviedro, fallas con estilolitos se indican a trazos y puntos y la dirección del movimiento está indicada con una cruz en aspa. En el diagrama correspondiente al O y $\mathrm{N}$ de Lorca las fallas inversas se indican por una línea gruesa a trazos (sus estrías con un asterisco). Con una estrella se indica la posición de $\sigma_{1}$, mientras $\sigma_{2}$ se indica con un triángulo y $\sigma_{3}$ con un cuadrado (En Murviedro los signos rellenos de mayor tamaño corresponden a la fase 1 , sin rellenar a la fase 2 y en pequeño tamaño y rellenos a la fase 3 ).

Para la deducida tan solo a partir de 6 fallas el resultado es: $\sigma_{1}=\mathrm{N} 83$, con un buzamiento de $58^{\circ}, \sigma_{2}=\mathrm{N} 332^{\circ}$ y buzamiento de $12^{\circ}$, y $\sigma_{3}=\mathrm{N} 235$ y $29^{\circ}$ de buzamiento. La razón áxica es 0,25 ; se trata del elipsoide de esfuerzos más prolato de todos.

Se ha realizado otra determinación de paleoesfuerzos. En este caso se usan las medidas obtenidas en el borde que limita la cuenca de Lorca y en la parte norte de Lorca, sin rebasar la autovía. También de allí se tienen medidas congruentes con las anteriores, si bien no se han usado estas últimas por no utilizar un área demasiado amplia. El total de medidas es de 28 (varias de ellas de fallas inversas) y su resultado (Fig. 5 y tabla 1) da una fase de deformación cuyos datos son: $\sigma_{1}=2^{\circ}$, con un buzamiento de $2^{\circ}, \sigma_{2}=\mathrm{N} 106^{\circ}$ y buzamiento de $82^{\circ}$, y $\sigma_{3}=\mathrm{N} 272$ y $8^{\circ}$ de buzamiento. La razón áxica es 0,36 ; se trata también de un elipsoide de esfuerzos prolato.

Un rasgo interesante son las fallas inversas de dirección aproximada E-O y vergencia al norte que afectan a las calcarenitas subverticales del borde sur de la cuenca de Lorca. Los desplazamientos de estas fallas producen unos resaltes en las calcarenitas cuyo tamaño oscila desde pocos centímetros a varios metros, generalmente de unas decenas de centímetros a un metro (valores mayores no serían visibles mucho tiempo pues los resaltes formados tenderían a caer al poco tiempo de su 
exposición). Tienen asociados uno o varios juegos de estrías congruentes con un movimiento inverso, en algunas ocasiones con ligera componente sinistrorsa. Además de ese rasgo se observa que, en algunos puntos, estas fallas inversas aumentan su buzamiento hacia uno de sus extremos y pasan a ser una falla de desgarre (Fig. 6A). En todos los casos en que se ha visto este hecho, el paso es a una falla de desgarre dextrorsa de dirección aproximada NNO-SSE, con estrías prácticamente horizontales, bien definidas. Se suele observar bien la zona de "giro" de falla inversa a falla vertical dextrorsa (o a la inversa).

También en el borde sur de la cuenca de Lorca se ha observado otro rasgo que muestra claramente que ha estado sujeto a compresión (Fig. 6B). El muro de las calcarenitas, que ahí buza unos $60^{\circ}$ al norte, está cortado por una falla inversa vergente al norte que hace cabalgar a sedimentos neógenos inferiores sobre las calcarenitas. Esta estructura es equivalente a las fallas anteriores que se acaban de describir. Además, en el techo de las calcarenitas, varios metros al norte de la falla, se ha desarrollado un "close-jointing" con neta vergencia opuesta. Ambos rasgos son compatibles con una compresión aproximadamente N-S y muestran la compresión a la que ha estado sometido ese borde. Naturalmente, la falla inversa que hace cabalgar al basamento sobre la serie neógena basal de la cuenca de Lorca (Fig.2) a lo largo de aproximadamente un kilómetro es igualmente congruente con esa compresión.

Dentro de la cuenca de Lorca, los yesos messinienses y las laminitas que existen en su base muestran localmente algunas estructuras menores. El mejor sitio donde se han observado está en el comienzo de la subida a la Quinquilla, cerca del río (Fig. 2). Allí corresponden sobre todo a pliegues, de tamaño centimétrico a métrico, cuya dirección media es, según las medidas realizadas, $\mathrm{N} 36^{\circ} \mathrm{E}$. Los ejes de estos pliegues buzan poco o son horizontales y las vergencias son tanto al E como al O. Todos estos pliegues se pueden interpretar como subsidiarios de un despegue de mayor tamaño en la dirección $\mathrm{N} 120^{\circ} \mathrm{E}$, situado en la base de un grueso paquete de yesos.

Unos $3 \mathrm{~km}$ más al norte, también en los yesos y en las laminitas, en La Serrata (Fig. 2), se observan pliegues y despegues. En este caso el desplazamiento de los materiales del techo, fundamentalmente los yesos, es hacia el OSO y parecen corresponder a deslizamientos gravitatorios. 

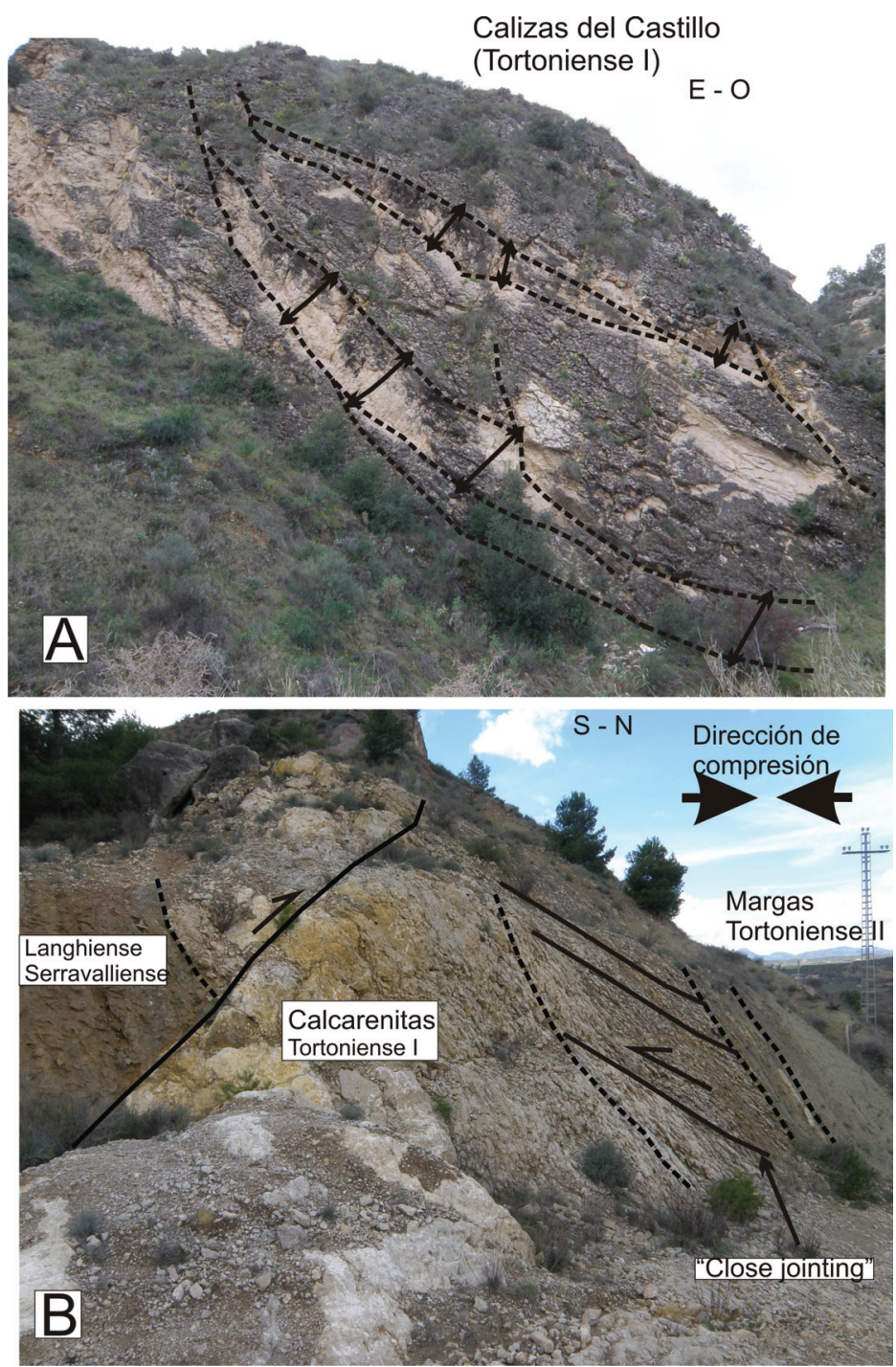

Fig 6. Borde sureste de la cuenca de Lorca con las calcarenitas fuertemente buzantes al N. A: Paso de fallas inversas a desgarres dextrorsos en calcarenitas con un buzamiento de unos $75^{\circ}$ al N. La pared visible tiene unos $25 \mathrm{~m}$ de anchura. Se marca la posición de algunas fallas inversas y de desgarre, pero se pueden ver más. El desplazamiento de las fallas se indica con líneas a trazos y doble flechas. B. Falla inversa vergente al N (situada a la izquierda) y diaclasación, a modo de close jointing, en lado N. Esta diaclasación es de vergencia opuesta a la de la falla y se desarrolla a techo de las calcarenitas, en su paso a las margas. Ambos rasgos son congruentes con una compresión N-S. El espesor de las calcarenitas es de unos $10 \mathrm{~m}$. 


\section{Discusión e interpretaciones}

El estudio de paleoesfuerzos muestra claramente una compresión de dirección NNO-SSE a N-S, prácticamente horizontal, y una extensión perpendicular que igualmente oscila entre las direcciones $\mathrm{N}^{\circ} 0^{\circ} \mathrm{E}$ y E-O aproximadamente. Este resultado es congruente con la existencia de las importantes fallas sinistrorsas de la región, de dirección general NNE-SSO a NE-SO. Los elipsoides resultantes son prolatos, lo que indica una neta diferencia entre los valores de los ejes principales. Esto, junto con el hecho de que el eje $\sigma_{2}$ se sitúa casi vertical, corresponde a una situación típica de tectónica de desgarre.

Hay sin embargo rasgos que permiten pensar que esos paleoesfuerzos se han relajado en algunos momentos (véanse las fases de menor importancia, la 2 y 3 de Murviedro en tabla 1 y Fig. 5) señaladas en el apartado de estructuras menores). Estas fases indican la existencia de momentos en los que $\sigma_{1}$ se aproxima a la vertical (valores de 50 y 58 grados) y $\sigma_{2}$ es el que pasa a ocupar aproximadamente la posición de $\sigma_{1}$ en el elipsoide principal de esfuerzos, mientras que $\sigma_{3}$ se mantiene prácticamente en la dirección N70 a E-O. Es decir, en algunos momentos ha habido un intercambio prácticamente completo entre los ejes $\sigma_{1}$ y $\sigma_{2}$ del elipsoide de esfuerzos, lo que como es natural, puede hacer que el movimiento de algunas fallas cambie, particularmente aquellas de dirección aproximadamente N-S que pueden entonces presentar movimientos de falla normal, perpendicular a la dirección de tensión N70 a E-O. Este rasgo de intercambio de ejes en la Cordillera Bética ya ha sido citado en diversas ocasiones (Sanz de Galdeano et al., 2010).

La dirección obtenida del paleoesfuerzo principal y de fases secundarias es muy parecida a la de anteriores resultados publicados por Armijo (1977), Bousquet y Philip (1976), Rutter et al. (1986) y Martínez Díaz (2002) para el sector comprendido entre Lorca y Totana. En particular, el último autor citado indica momentos de extensión, que pueden ser interpretados del modo que se acaba de señalar en Sanz de Galdeano et al. (2010) (también explica esto la existencia de terremotos con mecanismos focales propios de fallas normales; Mezcua et al., 1984).

Los esfuerzos deducidos son los que han controlado las grandes fallas, las cuales a su vez en el sector de Lorca han modelado desde finales del Mioceno medio a la actualidad la terminación NE de la sierra de las Estancias (límite SE de la cuenca de Lorca). Como resultado de ello, los sedimentos neógenos de dicho límite buzan fuertemente hacia el norte o incluso localmente están verticalizados y cabalgados por el basamento.

Ese límite se encuentra afectado en numerosos puntos por fallas que lo cortan transversalmente y le producen irregularidades que hacen perder su continuidad. Estos rasgos sirven también para descartar la existencia de una falla longitudinal en esa posición. Además, las fallas inversas antes citadas, el "close jointing" desarrollado en calcarenitas y el paso de las fallas inversas a las de desgarre, no indican ni movimientos normales ni transcurrentes dextrorsos a lo largo de ese límite. La falla que afecta a la base de las calcarenitas aproximadamente a $1 \mathrm{~km}$ al oeste del Castillo con movimientos sinistrorsos puede ser interpretada como formada a causa de la 
diferente competencia de los materiales, empujados por los desplazamientos de la FAM.

Cabría pensar en la posibilidad de la existencia de una falla bajo el Guadalentín paralela al borde sur de la cuenca de Lorca, es decir, de dirección aproximadamente E-O, y que no fuera visible. Pero en superficie desplazaría a los niveles de yesos que hay al norte del Cejo de los Enamorados (dado que el movimiento sería transversal a las capas), lo que no se observa. Igualmente, la hipotética falla de Las Viñas habría producido un desplazamiento dextrorso de los niveles de yesos, que sin embargo no se observa. Tan solo se observa que directamente al $\mathrm{N}$ del Cejo de los Enamorados los yesos se acercan al borde sur de la cuenca, de manera que parece que el espesor de limos y margas existente entre las calcarenitas y los yesos es menor ahí que, por ejemplo, al este de La Serrata. Este cambio de espesor puede deberse a la tectónica que constantemente ha afectado el borde sur de la cuenca de Lorca, en concreto al desplazamiento de la falla NNE-SSO del Cejo de los Enamorados en su prolongación hacia el NNE. A su vez, esta prolongación que geométricamente es obligada, va también en contra de la posible falla situada bajo el Guadalentín.

Las estructuras antes descritas en los yesos y laminitas también están netamente relacionadas con la tectónica que afecta a la región. En las del sector de la Quinquilla, se pueden interpretar como ligadas al movimiento de la falla del Cejo de los Enamorados, producidas durante el desplazamiento hacia el SSO del bloque occidental de esa falla. En las de la Serrata, interpretadas como debidas a un proceso gravitatorio, pudieron formarse simplemente durante el levantamiento regional de grandes zonas de las Béticas, la de Lorca incluida, aunque no esta no sea la región donde el levantamiento ha sido más importante (Sanz de Galdeano y Alfaro, 2004).

Las estructuras del borde sur de la cuenca de Lorca se inscriben en el marco de las deformaciones producidas por las fallas principales que se prolongan desde el NE de Murcia a Puerto Lumbreras, e incluso a Alborán. En ese contexto, la elevación de la parte NE de la sierra de las Estancias es congruente con la compresión casi N-S que afectó a toda la zona. Ahí produjo un antiforme, mientras que en el borde sur de la propia cuenca de Lorca se formó un sinforme.

En este marco regional, la falla próxima al Cejo de los Enamorados continúa más al NNE, dentro de la propia cuenca de Lorca, y es paralela a las fallas principales, lo que indica que la zona afectada por dichas fallas es muy amplia.

\section{Conclusiones}

El estudio del sector de Lorca muestra el hecho, previamente conocido, de que la falla de Alhama de Murcia está dividida en numerosas líneas paralelas que en su conjunto forman una amplia zona de falla. Además de ellas, hay fallas paralelas que han de considerarse como pertenecientes a este juego de fallas y que se sitúan a varios kilómetros de distancia, tal como es la falla del Cejo de los Enamorados o las que hay bajo la depresión del Guadalentín. Existen otras estructuras asociadas importantes, como es el antiforme de la sierra de las Estancias en el área de Lorca. 
La sierra de las Estancias comenzó a elevarse en las cercanías de Lorca desde inicios del Mioceno superior, incluso desde el Serravalliense. Sus bordes, en particular el NE, a la vez borde SE de la cuenca de Lorca, muestran un levantamiento progresivo. Esto se deduce a partir de la litología de los materiales previamente erosionados y redepositados y por los muy diferentes espesores de los sedimentos neógenos, particularmente hasta las calcarenitas del Castillo, aunque también los términos más altos demuestran esa elevación progresiva.

La creación del antiforme del castillo de Lorca está en directa relación con el movimiento de las grandes fallas de desgarre. Su flanco norte es el borde NE de la sierra de las Estancias y el flanco SE se formó por la acción directa de la FAM. Es naturalmente congruente con los paleoesfuerzos obtenidos.

También es congruente con dichos paleoesfuerzos el conjunto de fallas transversales, grandes y pequeñas, que afectan al borde NE de la sierra de las Estancias, tanto las fallas de desgarre como las inversas y las que combinan ambos rasgos de fallas de desgarre e inversas.

Esas estructuras, entre las que destaca la importante falla del Cejo de los Enamorados, descartan la existencia de una falla E-O normal o de desgarre en el borde NE de la sierra de las Estancias. También parece descartarse la existencia de una falla paralela bajo el Guadalentín, en ese borde SE de la cuenca de Lorca.

Los paleoesfuerzos muestran sobre todo una compresión N-S a NNO-SSE y una extensión perpendicular, que determinan el funcionamiento sinistrorso de la FAM y dextrorso de las fallas conjugadas de dirección NO-SE. A la vez indican que en algunos momentos se produjo un intercambio entre los ejes $\sigma_{1}$ y $\sigma_{2}$, lo que permite, particularmente en fallas de dirección casi N-S, desplazamientos de tipo normal y por tanto la posibilidad de ese tipo de mecanismos focales en algunos terremotos.

\section{Agradecimientos}

Agradecemos a dos revisores anónimos sus sugerencias y correcciones que han permitido mejorar la calidad del artículo. Este estudio es una contribución de los proyectos TOPO-IBERIA CONSOLIDER-INGENIO (CSD2006-00041), CGL2009-11308 del Ministerio de Ciencia e Innovación, RNM-5388 y RNM-370 (Junta de Andalucía). Angel Caballero (IACT) y Sergio Hernández (UPV-EHU) dibujaron parte de las figuras. Alejandro Robador (IGME) facilitó amablemente información de subsuelo de la cuenca del Guadalentín.

\section{Referencias bibliográficas}

ARMIJO, R. (1977). La zone des failles Lorca-Totana (Cordillères Bétiques, Espagne). Etude tectonique et neotectonique. MsC Thesis, University, Paris VII, $229 \mathrm{p}$.

BOOTH REA, G. \& AZAÑÓN, J. M. (2003). "Extensión versus compresión durante el Mioceno Superior en la Cuenca de Lorca (Béticas orientales)". Geogaceta, 34, 83-86.

BOOTH REA, G. \& SILVA, P. (2010). Mapa Geológico de España E 1:50.000, Hoja $n^{\circ} 953$ (Lorca), Cartografía y Memoria, 195 p. 
BOUSQUET, J. C. (1979). "Strike-slip faults in southeastern Spain". Tectonophysics, 52, 277-286.

BOUSQUET, J. C. \& MONTENAT C. (1974). "Présence de décrochements nordest-sud-ouest plio-quaternaires dans les Cordillères Bétiques orientales (Espagne)". Comptes rendues Académie des Sciences, 278, 2617-2620.

BOUSQUET J.C. \& PHILIP H. (1976). Observations microtectoniques sur la compression nord-sud quaternaire des Cordillères bétiques orientales (Espagne méridionale-Arc de Gibraltar). Bulletin de la Societé géologique de France, 18, 711-724.

DINARÈS TURELL, J., SPROVIERI, R., CARUSO, A., GOMIS-COLL, E., DI STEFANO, E., PUEYO, J.J., ROUCHY, J.M. \& TABERNER, C. (1998). Preliminary integrated magnetostratigraphic and biostratigraphic correlation in the Miocene Lorca Basin (Murcia, SE Spain). Acta Geologica Hispanica, 34, 339351.

GALINDO-ZALDÍVAR, J. \& GONZÁLEZ-LODEIRO, F. (1988). Faulting phase differentiation by means of computer search on a grid pattern. Annales Tectonicae, II, 2: 90-97.

GARCÍA MAYORDOMO, J. (2005). Caracterización y análisis de la peligrosidad sísmica en el sureste de España. Tesis Doctoral, Univ. Complutense de Madrid, $373 \mathrm{p}$.

GAUYAU, F. (1977). Étude géophysique dans le Levant Espagnol (entre Alicante et Totana): le problème du prolongement de l'accident d'Alhama de Murcia. Thèse. Université des Sciences et Techniques du Languedoc. Montpellier, $129 \mathrm{p}$. GUILLÉN MONDÉJAR F., RODRÍGUEZ ESTRELLA, T., ARANA, R. \& LÓPEZ AGUAYO, F. 1995. Historia geológica de la cuenca de Lorca: influencia de la tectónica en la sedimentación. Geogaceta, 18, 30-33.

IGME (1976). Cortes geoeléctricos. Prospección geofísica en el alto Guadalentín. Plan sectorial de aguas subterráneas. Subsector Cuenca del Segura. Planos nº 28 у 29.

IGME (1982). Ampliación de la investigación de pizarras bituminosas en la zona de Lorca (Murcia), $166 \mathrm{p}$.

KAMPSCHUUR, W., LANGENBERG, C.W., RONDEEL, H.E., ESPEJO, J.A., CRESPO, A. \& PIGNATELLI, R. (1981). Mapa Geológico de España, e: 1:50.000, hoja 953 (Lorca). I.G.M.E., Memoria de 43 p.

LAROUZIĖRE, F. D. de, BOLZE, J., BORDET, P., HERNÁNDEZ, J., MONTENAT, C. \& OTT D'ESTEVOU, P. (1988). The Betic segment of the lithosperic Trans-Alboran shear zone during the Late Miocene. Tectonophysics, $152,41-52$.

MARTÍNEZ DÍAZ, J. J. (1998). Neotectónica y Tectónica Activa del sector centrooccidental de la región de Murcia y sur de Almería. 2 tomos. Tesis Doctoral, Univ. Complutense Madrid, $466 \mathrm{p}$.

MARTÍNEZ DÍAZ, J. J. (1999). Sismotectónica de la falla de Alhama de Murcia. Implicaciones sismogenéticas del terremoto de Lorca de Junio-1977. (Mb: 4.2). Estudios Geológicos, 55, 251-266. 
MARTÍNEZ DÍAZ, J. J. (2000). Indicios geomorfológicos indicativos de tectónica activa en el sector Lorca-Totana de la falla de Alhama de Murcia. Boletín Geológico y Minero, 111, 67-76.

MARTÍNEZ DÍAZ, J. J. (2002). Stress field variation related to fault interaction in a reverse oblique-slip fault: the Alhama de Murcia fault, Betic Cordillera, Spain. Tectonophysics, 356, 1/3, 291-305.

MARTÍNEZ DÍAZ, J. J. \& HERNÁNDEZ ENRILE, J. L. (1991). Reactivación de la Falla de Alhama de Murcia (sector de Lorca-Totana): Cinemática y campos de esfuerzos desde el Messiniense hasta la Actualidad. Geogaceta, 9, 38-42.

MARTÍNEZ-DÍAZ, J.J., MASANA, E. \& Ortuño, M. (2012). Active tectonics of the Alhama de Murcia fault, Betic Cordillera, Spain. Journal of Iberian Geology, 38, 269-286, doi:10.5209/rev_JIGE.2012.v38 .n1.39218.

MEZCUA, J., HERRAIZ, M. \& Buforn E. (1984). Study of the 6 June 1977 Lorca (Spain) earthquake and its aftershock sequence. Bulletin of the Seismological Society of America, 74, 169-179.

MONTENAT, C., OTT D’ESTEVOU, P. \& DELORT, T. (1990). Le Bassin de Lorca.. Documents et Travaux de l'IGAL, 12-13, 261-280.

PÉREZ LORENTE, F., RODRÍGUEZ ESTRELLA, T., SERRANO, F., MANCHEÑO, M.A., GUILLÉN MONDÉJAR, F., ARANA, R., LÓPEZAGUAYO, F., \& FERNÁNDEZ TAPIA, M.T. (1992). Contribución a la bioestratigrafía neógena de la Cuenca de Lorca (Murcia). III Congreso Geológico de España y VIII Congreso Latinoamericano de Geología, Salamanca. Actas Tomo 1, 185-190.

REY PASTOR, A. (1951). Estudio sismotectónico de la región Sureste de España. Instituto geográfico y Catastral. Madrid, $23 \mathrm{p}$.

ROUCHY, J.M., TABERNER, C., BLANCVALLERON, M.M., SPROVIERI, R., RUSSELl, M., PIERRE, C., DI STEFANO, E., PUEYO, J. J., CARUSO, A., DINARÈS TURELL, J., GOMISCOLL, E., WOLFF, G. A., CESPUGLIO, G., DTCHFIELD, P., PESTREA, S., COMBORIEUENEBOUT, N., SANTISTEBAN, C., \& GRIMALT, J. O. (1998). Sedimentary and Diagenetic Markers of the Restriction in a Marine Basin - The Lorca Basin (SE Spain) During the Messinian. Sedimentary. Geology, 121 (1-2), 23-55

RUTTER, E.H., MADDOCK, R.H., HALL, S.H., WHITE, S. (1986). Comparative microstructures of natural and experimentally produced clay-bearing fault gouges. Pure and Applied Geophysics, 124 (1-2), $1-30$.

SANZ DE GALDEANO, C. \& ALFARO, P. (2004). Tectonic significance of the present relief of the Betic Cordillera. Geomorphology, 63, 178-190.

SANZ DE GALDEANO, C., SHANOV, S., GALINDO ZALDÍVAR J., RADULOV, A. \& NIKOLOV, G. (2010). A new tectonic discontinuity in the Betic Cordillera deduced from active tectonics and seismicity in the Tabernas Basin. Journal of Geodynamics, 50, 57-66. DOI: 10.1016/j.jog.2010.02.005. 
SANZ DE GALDEANO, C. \& VERA, J.A. (1992). Stratigraphic record and palaeogeographical context of the Neogene basins in the Betic Cordillera, Spain. Basin Research, 4, 21-36.

SILVA, P., GOY, J.L., \& ZAZO, C. (1992). Características estructurales y geométricas de la falla de desgarre de Lorca-Alhama. Geogaceta. 12, 7-10.

SILVA, P.G., GOY, J.L., ZAZO, C. \& BARDAJÍ, T. (2003). Fault-generated mountain fronts in southeast Spain: Geomorphologic assessment of tectonic and seismic activity. Geomorphology, 50, 203-225, doi:10.1016/S0169$555 \mathrm{X}(02) 00215-5$. 